\title{
Widerrufbarkeit der Einwilligung
}

Der Grundsatz der freien Widerrufbarkeit der Einwilligung ist im Datenschutzrecht seit jeher allgemein anerkannt und unter Geltung der DS-GVO mittlerweile auch in deren Art. 7 Abs. 3 ausdrücklich normiert. So sinnvoll und wichtig dieser Grundsatz im Sinne einer effektiven informationellen Selbstbestimmung ist, so schwierig sind teilweise die Konsequenzen zu handhaben, die mit diesem Grundsatz einhergehen, etwa im Forschungskontext oder bei der Einbettung der Einwilligung in ein umfassenderes vertragliches Austauschverhältnis.

\section{Ausgangspunkt}

Nach Art. 7 Abs. 3 S. 1 DS-GVO hat die betroffene Person „das Recht, ihre Einwilligung jederzeit zu widerrufen“. Die freie Widerrufbarkeit der Einwilligung erlaubt es dem Einzelnen, eine Entscheidung über die Preisgabe personenbezogener Daten nachträglich noch einmal zu korrigieren. Wichtig ist ein solches Korrekturrecht vor allem deshalb, weil es für den Einzelnen in Zeiten von Big Data zunehmend schwierig oder gar unmöglich ist, die langfristigen Konsequenzen und die ganze Tragweite einer einmal erteilten Einwilligung zu überblicken und realistisch einzuschätzen. Bestünde hier keine Möglichkeit, eine einmal getroffene Entscheidung nachträglich wieder zu revidieren, würde dies letztlich zu einer Aushöhlung des Grundrechts auf informationelle Selbstbestimmung führen. ${ }^{1}$ Entsprechend ist es auch nicht möglich, dass der Einzelne auf sein Widerrufsrecht verzichtet, auch dies wäre mit dem informationellen Selbstbestimmungsrecht nicht vereinbar und eine dahingehende Klausel unwirksam. ${ }^{2}$

$\mathrm{Zu}$ den Rechtsfolgen des Widerrufs einer Einwilligung findet sich in Art. 7 Abs. 3 S. 2 DS-GVO eine Regelung. Danach wird die Rechtmäßigkeit der aufgrund der Einwilligung bis zum Widerruf erfolgten Verarbeitung durch den Widerruf nicht berührt. Unzulässig ist jede weitere Datenverarbeitung für die Zukunft. Nach Art. 17 Abs. 1 lit. b DS-GVO hat die betroffene Person zudem das Recht, von dem für die Verarbeitung Verantwortlichen die Löschung der sie betreffenden Daten zu verlangen, und der für die Verarbeitung Verantwortliche ist verpflichtet, diese Daten unverzüglich zu löschen. ${ }^{3}$

\section{Forschung}

Forschung bedarf, zumindest wenn sie langfristig angelegt ist, einer verlässlichen Datenbasis. Der Grundsatz der freien Widerrufbarkeit einer Einwilligung lässt sich mit diesem Bedürfnis nur schwer vereinbaren, muss doch stets damit gerechnet werden, dass einer Forschung mit personenbezogenen Daten die Legitimationsgrundlage wieder entzogen wird und diese daher im bisherigen Zuschnitt nicht mehr fortgesetzt werden kann. Gleichwohl gilt der Grundsatz der freien Widerrufbarkeit einer Einwilligung auch im Forschungskontext, insoweit sieht das Daten-

1 Buchner/Kühling in Kühling/Buchner, DS-GVO/BDSG (3. A. 2020), Art. 7 Rn. 34.

2 S. schon Herbst MedR 2009, 149 (150).

3 Zu möglichen Ausnahmen s. Art. 17 Abs. 1 lit. b a. E. und Abs. 3 DS-GVO. schutzrecht keine Privilegierung der Forschungsdatenverarbeitung vor. Lediglich punktuell sind enge Ausnahmen normiert, etwa für klinische Prüfungen im Arzneimittelrecht (s. dazu $\$ 40$ Abs. 2a S. 2 Nr. 3 AMG).

Unklar ist auch, wie mit dem Grundsatz der freien Widerrufbarkeit der Einwilligung bei der sog. Datenspende umgegangen werden soll. Einer solchen Daten-,Spende“, wie sie u.a. der Ethikrat vorgeschlagen hat, um eine Datennutzung ohne enge Zweckbindung zugunsten der klinischen und medizinbezogenen Grundlagenforschung zu ermöglichen, ${ }^{4}$ liegt an sich die Idee einer dauerhaften und unwiderruflichen Hingabe von Daten zugrunde. Mit dem geltenden Datenschutzrecht, insbesondere in Gestalt des Art. 7 Abs. 3 DSGVO, lässt sich diese Idee allerdings nicht vereinbaren. Konsequenterweise ist daher auch die „Datenspende " nach dem Patienten-Datenschutz-Gesetz (PDSG) widerruflich ausgestaltet ${ }^{5}$ - und damit gerade keine „Spende“ im Sinne einer freiwilligen und grundsätzlich unwiderruflichen Hingabe eines Spendengegenstands. ${ }^{6}$

\section{Vertragliche Einbettung der Einwilligung}

Problematisiert wird der Grundsatz der freien Widerrufbarkeit einer Einwilligung auch in Konstellationen, in denen die Einwilligung im Rahmen eines umfassenderen Vertragsverhältnisses erteilt wird oder sogar unverzichtbare Voraussetzung für ein solches Vertragsverhältnis ist. ${ }^{7}$ Teils gehen die Forderungen hier dahin, auch bei der Frage der Widerrufbarkeit diese rechtsgeschäftliche Einbindung der Einwilligung zu berücksichtigen - mit der Konsequenz, dass eine Einwilligung, wenn sie Gegenstand eines Vertrags ist, nicht einfach einseitig wieder rückgängig gemacht werden kann. Alternativ wird gefordert, in solcherlei Fällen einen vertraglichen Ausschluss der freien Widerrufbarkeit der Einwilligung zuzulassen.

Allerdings gilt auch hier wieder, wie schon für die Forschungsdatenverarbeitung, dass Art. 7 Abs. 3 DS-GVO eine solche wertende Einschränkung der freien Widerrufbarkeit der Einwilligung nicht zulässt. Zu berücksichtigen ist insoweit auch der Erwägungsgrund 42 zur DS-GVO, in dessen Satz 5 betont wird, dass die betroffene Person in der Lage sein muss, ihre Einwilligung zurückzuziehen, „ohne Nachteile zu erleiden“. Auch mit dieser Wertung ist eine Einschränkung der freien Widerrufbarkeit der Einwilligung nur schwer vereinbar. Letztlich bleibt daher in Konstellationen, in denen eine Einwilligung in die Datenverarbeitung an sich zentrales Element eines Vertragsverhältnisses ist, diese dann aber gleichwohl widerrufen wird, nur der Ausweg, alternativ auf den Erlaubnistatbestand der Vertragserfüllung nach Art. 6 Abs. 1 lit. b DS-GVO zurückzugreifen. ${ }^{8}$

\footnotetext{
4 Deutscher Ethikrat, Big Data und Gesundheit - Datensouveränität als informationelle Freiheitsgestaltung (2017), S. $266 \mathrm{f}$.

5 Ausführlich dazu Dochow MedR 2021, 115 (117 ff.)

6 S. in diesem Sinne von Ulmenstein PinG 2020, 47.

7 Vgl. Buchner/Kühling in Kühling/Buchner, DS-GVO/BDSG (3. A. 2020), Art. 7

8 Zu den Voraussetzungen hierfür im Einzelnen s. Buchner/Kühling in Kühling/Buchner, DS-GVO/BDSG (3. A. 2020), Art. 7 Rn. 39a.
} Rn. $38 \mathrm{ff}$. 